\title{
In vivo detection of external apical root resorption induced by apical periodontitis using periapical radiography and cone-beam computed tomography
}

\section{Stephanie Diaz Huamán}

University of Sao Paulo: Universidade de Sao Paulo

Maya Fernanda Manfrin Arnez

Universidade de Sao Paulo

Fernanda Maria Machado Pereira Cabral de Oliveira

Universidade de Sao Paulo

Andiara De Rossi

Universidade de Sao Paulo

Léa Assed Bezerra Silva

Universidade de Sao Paulo

Francisco Wanderley G Paula-Silva ( $\nabla$ franciscogarcia@forp.usp.br)

Universidade de Sao Paulo https://orcid.org/0000-0001-8559-532X

\section{Research Article}

Keywords: Apical root resorption, periapical radiography, cone beam computed tomography, cementum, dentin

Posted Date: March 4th, 2021

DOl: https://doi.org/10.21203/rs.3.rs-173059/v1

License: @ (i) This work is licensed under a Creative Commons Attribution 4.0 International License.

Read Full License 


\section{Abstract}

Objectives: To investigate sensitivity, specificity, predictive values and accuracy of periapical radiography (PR) and Cone beam computed tomography (CBCT) for detection of external apical root resorption (EARR).

Materials and Methods: Dog's teeth with experimentally induced root resorption underwent or not root canal treatment ( $n=62$ roots). True positives (TP), false positives (FP), true negatives (TN) and false negatives (FN) in PR and CBCT diagnoses were determined using histopathologic findings as gold standard. Sensitivity, specificity, positive predictive value (PPV), negative predictive value (NPV) and diagnostic accuracy (TP + TN) in the diagnosis of EARR were calculated. Data was compared using chisquared test $(\mathbb{\nabla}=0.05)$.

Results: We found that PR detected EARR in 35\% of roots and CBCT, in 47\%. EARR was microscopically diagnosed in 50\% ( $p=0.03$ comparison between PR and microscopy; $p=0.67$ comparison between CBCT and microscopy). Overall, CBCT produced more accurate diagnoses than PR ( 0.93 for CBCT versus 0.70 for PR; $p=0.008$ ). Interestingly, when data was stratified into small and large resorptions, PR and CBCT allowed identification of large resorption in $100 \%$ of the cases and showed the same accuracy. However, for small resorptions, PR showed an accuracy of 0.83 , whereas CBCT showed an accuracy of $0.96(p=$ $0.003)$.

Conclusions: We demonstrated that CBCT showed higher accuracy to detect EARR. These findings shed light on the use of CBCT for detection of initial root resorption.

Clinical relevance: Early identification of resorption allows a prompt treatment and reduces the risk of dental structure loss.

\section{Introduction}

External apical root resorption (EARR) might be induced by endodontic pathogens in a pathologic process, consequence of a persistent stimulation of inflammatory mediators that triggers a resorptive mechanism, which gradually eliminates cementoblasts, cementum and the dentin area on the external surface of the dental root $(1,2)$.

Current technology has given access to new adjunct diagnostic tools to perform an adequate diagnosis. Though periapical radiography (PR) remains as one of the most used diagnostic aids in dentistry, Cone beam computed tomography (CBCT) are now available for the practitioner to deliver a better diagnostic and treatment plan. CBCT is a well-established method that compensate for the PR drawbacks such as distortion, anatomic superimposition and magnification, allowing the operator to completely explore the region of interest in a CBCT scan using a computer program $(3,4)$. 
Small field of view (FOV) cone beam computed tomography (CBCT) has increasingly replacing several radiological procedures (5). In cases in which lower dose radiography does not provide adequate or satisfactory diagnostic information, small field of view CBCT has been recommended for assessment and/or management of root resorption when imaging would potentially change or enhance treatment plan (6).

Previously we demonstrated that cone beam computed tomography (CBCT) allows detection of small size apical periodontitis (AP) and that healing rates following root canal treatment were lower than previous radiographic studies have predicted (7-9). Specifically, for investigation of external root resorption, CBCT has a high accuracy in detecting ex-vivo artificial dental root surface mineral loss (1013). Even though previous demonstrated that CBCT portrays an attractive and valuable option to assess periodontal and periapical tissues, its accuracy to detect external root resorption in vivo has not been investigated. Therefore, this study aims to investigate sensitivity, specificity, predictive values and accuracy of periapical radiography (PR) and Cone Beam Computed Tomography (CBCT) in diagnosing external apical root resorption.

\section{Materials And Methods}

This is a secondary study based on experimental protocols and material generated by the study of PaulaSilva et al. $(7,8)$, previously approved by the Animal Research Ethical Committee of Ribeirão Preto Dental School of the University of São Paulo (process number 07.1.192.53.6). Briefly, an animal experiment with 12 mongrel dogs ( 12 months of age, body weight from 10 to $15 \mathrm{~kg}$ ) was performed and, for that, premolar teeth were used to induce AP (62 dental roots of dogs' teeth). The animals received medication (Drontal Puppy Bayer, São Paulo, SP), vitamins (Glicopan Pet; Vetnil Indústria e Comércio de Produtos Veterinarios Ltda, Louveira, SP), and vaccines (Rai-Vac l; Fort; Vanguard HTLP 5 / CV-L; Laboratórios Pfizer Ltda., Guarulhos, SP). The dogs were kept in the vivarium at the School of Dentistry of Ribeirão Preto at the University of São Paulo with free access to water and standardized diet. Dogs were anesthetized and sedated previous every procedure.

Roots with AP were treated either by one-visit, two-visit therapy or left untreated. The animals were followed throughout the experimental period to observe changes in eating habits, development of inflammatory processes or suppuration of tissues, among other abnormalities. After 6 months, the animals were euthanized with a lethal intravenous overdose of sodium pentobarbital. The mandibles were dissected and sectioned to obtain individual roots that were fixed in $10 \%$ buffered formalin for 72 hours, demineralized in EDTA, and embedded in paraffin. The specimens were serially sectioned. Longitudinal sections of 5-mm thickness were stained with hematoxylin and eosin (HE).

Periapical radiographs used in this study was performed according to the parallelism technique, using a device for standardizing radiographic takes in dogs (14). Ultraspeed® size 2 periapical films (Eastman Kodak Company, Rochester, USA), Heliodent ${ }^{\circledR}$ dental X-ray machine (Siemens, New York, USA), with 60 $\mathrm{kVp}$ and $10 \mathrm{~mA}$ and exposure time of 1 second were used. The radiographs were developed manually 
using the time / temperature method and filed in plastic cards. The images were digitalized using an optical scanner (Scanjet 7450C, Hewlett-Packard; Palo Alto, CA) with a resolution of 1,200 dpi.

CBCT scans were performed using a NewTom $3 \mathrm{G}$ cone beam computed tomography equipment (QR Srl, Verona, Italy). For this study, $120 \mathrm{kv}$ and $3.6 \mathrm{~mA}$ were used for standardization of the technique, with 9inch FOV (field of view) and exposure time of 36 seconds. The NewTom $3 G$ device captures 360 images with an interval of 1 between them, in 36 seconds, with an image reconstruction resolution of $512 \times 512$ pixels and 12 bits per pixel (4096 gray scale).

To investigate the presence of EARR in the current report, microscopic slides from 62 roots were collected and examined under fluorescence microscopy using HE stained cuts (excitation at 460-500 nm and emission at 512-542 $\mathrm{nm}$ ) at $20 \times$ magnification. A skilled and trained observer classified EARR as small external apical root resorption when mineralized tissue loss involved cementum only or large external root resorption when mineralized tissue loss involved cementum and dentin.

Three calibrated examiners $(\mathrm{K}=0.94)$ evaluated presence of external root resorption in radiographic and tomographic images. For radiographic analysis, images were assessed using the Image J 1.28 Software (National Institutes of Health, Bethesda, MD) in which brightness, contrast and magnification tools could be used. For CBCT evaluation, the NewTom 3G equipment software was used. In order to allow a direct comparison with the periapical radiography, the use of sections of 1 millimeter thick with 0.5 millimeter spacing between sections was standardized, in sagittal view.

Based on the presence of microscopically detected small external root resorption (involving cementum only) or large external root resorption (involving cementum and dentin), true-positives (TPs), falsepositives (FPs), true-negatives (TNs), and false-negatives (FNs) in PR and CBCT diagnoses were determined using microscopy findings as the gold standard. Sensitivity, specificity, positive predictive value (PPV), negative predictive value (NPV), and diagnostic accuracy (true positives + true negatives) in the diagnosis of AP were calculated. Data was compared using chi-squared test $(\mathbb{\nabla}=0.05)$.

\section{Results}

After microscopic analyses, we found that external root resorption was present in $50 \%$ of the specimens. PR allowed detection of EARR in $35.5 \%$ of roots and CBCT in $46.8 \%$. ( $p=0.03$ comparison between PR and microscopy; $p=0.67$ comparison between CBCT and microscopy) (Table 1). False negative diagnoses were most common after PR evaluation ( $14.5 \%$ of the roots) than CBCT ( $3.2 \%$ of the roots). Both tools presented adequate specificity, yet CBCT presented higher sensitivity (0.93). Results of TP, FP, TN, FN, sensitivity, specificity, PPV, NPV, and diagnostic accuracy for diagnosis of EARR are summarized in Tables 2 and 3. CBCT produced more accurate diagnoses than PR $(p=0.008)$.

When data was stratified into small and large resorptions, we found that for large resorptions, in which microscopically cementum and dentin were damaged, PR and CBCT allowed identification of resorption in $100 \%$ of the cases and showed the same accuracy. For small resorptions, in which only cementum was 
damaged, PR showed an accuracy of 0.83 whereas CBCT showed an accuracy of $0.96(p=0.003)$. PR had a lower sensitivity $(0.62)$ in finding small resorptions, yet its positive predictive value was $100 \%$. Results of TP, FP, TN, FN, sensitivity, specificity, PPV, NPV, and diagnostic accuracy for diagnosis of small EARR and large resorptions are summarized in Tables 2 and 3.

\section{Discussion}

Our study showed that CBCT has a higher sensitivity in detecting external apical root resorption than PR. Nevertheless, both techniques presented a similar specificity. Our results are consistent with previous studies that reported CBCT as the most accurate method in finding artificial external root resorption when compared to panoramic or periapical radiography $(13,15,16)$. Even though, we observed that $P R$ has been found to be less sensitive, a high specificity of PR was obtained.

In order to obtain detailed information about the degree of mineralised tissue resoprtion, we classified large and small resorption by analyzing the affected mineralized tissue after histological processing. Large resorption, in which cementum and dentin were damaged, were detected using both radiographic techniques. For small resorption detection, however, where only cementum was affected, PR had a lower sensitivity than CBCT. These results could be explained by a previous study that stated that mineral bone loss could not be detected by the human eye in periapical radiograph until reaching $7,1 \%$ of loss (17), indicating that small resorption will not be perceive using conventional radiographic techniques, whereas CBCT will provide a better sensitivity and accuracy in detecting apical external root resorption.

In some cases, cone beam computed tomography (СBCT) has increasingly replacing conventional radiological procedures due to the possibility of arbitrary reconstructions and views free of superimposition (5). Previous studies have reported that CBCT has a high accuracy in detecting EER, however these studies were performed under different methodologies, in ex vivo models with artificial or natural EER, that may impair their results after comparison with other radiographic techniques (10-13). Our analysis was performed in teeth that were treated endodontically or not in vivo, in which soft tissue thickness as well as other anatomic features that may influence the periapical image are considered. Furthermore, using the microscopic analysis as gold standard assures an accurate diagnosis, as was previously reported by our research group (7).

Imaginologic evaluation is a diagnostic tool of irrefutable importance when needing further and careful assessment; however, previous detailed clinical evaluation should be performed in order to prescribe radiation-based imaginologic tools (18). Several international groups have published statements aiming to provide a recommendation about CBCT indication (19-21). The benefits of using CBCT are widely known and recognized, though the amount of radiation used each scanning time is still a matter of controversy. When in need of a detailed image, optimization of radiation exposure may be performed. In cases of impacted and supplementary teeth, dentoalveolar trauma, orofacial defects dental anomalies and bone pathologies, where there is a justified reason for CBCT prescription, effective dose should be managed (21). Reduction of radiation risk could be achieved by managing $X$-Ray tube voltage and current 
(22), exposure time, FOV, number of projections and patient's shielding devices (19). Considering that the amount of radiation is directly related to the period of image taken and the field of the vision (FOV) (23), diagnostic modalities with smaller field are currently used. Limited cone-beam computed tomography (LCBCT) offers a small FOV $(1.6-3.1 \mathrm{inch} / 4-8 \mathrm{~cm})$ with a high-resolution image with less radiation than a common CT $(24,25)$. In this study, a 9-inch FOV was used to acquire images of the entire low jaw of the animal because several teeth were used for investigation. Clinically, a smaller FOV could be used if we consider that a single tooth should be examined.

This study has compared CBCT and PR's accuracy in diagnosing EARR in an animal model. Even though an animal model is a more adequate subject of study than in vitro specimens, some limitations may arise such as species' difference and sample size. The canine specie has more similarities in perioral and oral tissues to humans than other species such as mice and guinea pigs, however, still exists differences such as teeth morphology that should be considered prior endodontic treatment. Furthermore, radiographs should be taken under complete anesthesia and with a pre-design standardization device to obtain a valuable image in all subjects. Another possible limitation could be our small sample size. Since this research was performed in animals, the sample was the minimum necessary that were needed to prove our hypothesis, following the technical regulations of the International Organization of Standardization. However, this limitation was considered in statistics analysis.

We found that CBCT was more accurate and sensitive when compared to PR in detecting external apical root resorption. These findings shed light on the use of $\mathrm{CBCT}$ for detection of initial root resorption that might occurs as a result of a dental trauma, pulp exposition or unmanaged orthodontic forces. Early identification of resorption allows a prompt treatment and reduces the risk of dental structure loss, nevertheless, CBCT should be prescribed under situations of strictly need and following effective dose optimization.

\section{Conclusions}

We demonstrated that CBCT showed higher accuracy to detect EARR. These findings shed light on the use of $\mathrm{CBCT}$ for detection of initial root resorption. Early identification of resorption allows a prompt treatment and reduces the risk of dental structure loss.

\section{Declarations}

Conflict of Interest: Author Stephanie Diaz Huamán declares that she has no conflict of interest. Author Maya Fernanda Manfrin Arnez declares that she has no conflict of interest. Author Fernanda Maria Machado Pereira Cabral de Oliveira declares that she has no conflict of interest. Author Andiara De Rossi declares that she has no conflict of interest. Author Léa Assed Bezerra Silva declares that she has no conflict of interest. Author Francisco Wanderley Garcia Paula-Silva declares that he has no conflict of interest. 
Funding: The work was supported by the Department of Pediatric Clinics, School of Dentistry of Ribeirão Preto, University of São Paulo, Ribeirão Preto, SP, Brazil, and São Paulo Research Foundation by a fellowship (06/51161-0) and grant (19/02060-7) to author Francisco Wanderley Garcia Paula-Silva and a grant (06/59072-7) to Léa Assed Bezerra da Silva.

Ethical approval: All applicable international, national, and/or institutional guidelines for the care and use of animals were followed. The study was approved by the Institutional Animal Research Ethics Committee (process \#07.1.192.53.6).

Informed consent: For this type of study, formal consent is not required.

\section{References}

1. Andreasen JO (1985) External root resorption: its implication in dental traumatology, paedodontics, periodontics, orthodontics and endodontics. Int Endod J 18(2):109-18.

2. Huang XX, Fu M, Hou BX (2019) Morphological Changes of the Root Apex in Permanent Teeth with Failed Endodontic Treatment. Chin J Dent Res 22(2):113-

3. Li F, Li J, Zhang D, Wu F (2018) Role of computed tomography scan in dental trauma: A crosssctional study. Dose Response 16(3):1559325818789837.

4. Shruthi N, Murthy BV, Sundaresh KJ, Mallikarjuna R (2013) Diagnosis demystified: CT as diagnostic tool in endodontics. BMJ Case Rep 2013:bcr2013010312.

5. Kaeppler G (2010) Applications of cone beam computed tomography in dental and oral medicine. Int J Comput Dent 13(3):203-19.

6. Patel S, Brown J, Semper M, Abella F, Mannocci F (2019) European society of endodontology position statement: Use of cone beam computed tomography in Endodontics: European Society of Endodontology (ESE) developed by. Int Endod J 52(12):1675-8.

7. de Paula-Silva FW, Wu MK, Leonardo MR, da Silva LA, Wesselink PR (2009) Accuracy of periapical radiography and cone-beam computed tomography scans in diagnosing apical periodontitis using histopathological findings as a gold standard. J Endod (35):1009-12.

8. de Paula-Silva FW, Santamaria M, Jr., Leonardo MR, Consolaro A, da Silva LA (2009) Cone-beam computerized tomographic, radiographic, and histologic evaluation of periapical repair in dogs' postendodontic treatment. Oral Surg Oral Med Oral Pathol Oral Radiol Endod 108(5):796-805.

9. de Castro R-MC, Maia-Filho E, Nelson-Filho P, Segato R, de Queiroz A, Paula-Silva F, et al. (2016) Single vs two-session root canal treatment: A preliminary randomized clinical study using cone beam computed tomography. J Contemp Dent Pract 17(7):515.

10. Vaz de Souza D, Schirru E, Mannocci F, Foschi F, Patel S (2017) External cervical resorption: A comparison of the diagnostic efficacy using 2 different cone-beam computed tomographic units and periapical radiographs. J Endod 43(1):121-5. 
11. Deliga Schroder AG, Westphalen FH, Schroder JC, Fernandes A, Ditzel Westphalen VP (2019) Accuracy of different imaging CBCT systems for the detection of natural external radicular resorption cavities: An ex vivo J Endod 45(6):761-7.

12. Goller Bulut D, Ugur Aydin Z (2019) The impact of different voxels and exposure parameters of CBCT for the assessment of external root resorptions: A phantom study. Aust Endod J 45(2):146-53.

13. Deliga Schroder AG, Westphalen FH, Schroder JC, Fernandes A, Westphalen VPD (2018) Accuracy of digital periapical radiography and cone-beam computed tomography for diagnosis of natural and simulated external root resorption. J Endod 44(7):1151-8.

14. Cordeiro RCL, Leonardo MR, Silva LABd, Cerri PS (1995) Desenvolvimento de um dispositivo para padronizaçäo de tomadas radiográficas em cä RPG, Rev Pós-Grad 2(3):138-40.

15. Heney CM, Arzi B, Kass PH, Hatcher DC, Verstraete FJM (2019) The diagnostic yield of dental radiography and cone-beam computed tomography for the identification of dentoalveolar lesions in cats. Front Vet Sci 6:42.

16. Takeshita WM, Chicarelli M, Iwaki LC (2015) Comparison of diagnostic accuracy of root perforation, external resorption and fractures using cone-beam computed tomography, panoramic radiography and conventional \& digital periapical radiography. Indian J Dent Res 26(6):619-26.

17. Bender IB (1982) Factors influencing the radiographic appearance of bony lesions. J Endod 8(4):16170.

18. Alimohammadi R (2018) Imaging of dentoalveolar and jaw trauma. Radiol Clin North Am 56(1):10524.

19. Commission E (2012) Radiation protection $n^{\circ} 172$, Cone beam CT for dental and maxillofacial Evidence-based guidelines.

20. Prescribing dental radiographs for infants, children, adolescents and individuals with special health care needs (2017) Pediatr Dent 39(6):205-7.

21. Oenning AC, Jacobs R, Pauwels R, Stratis A, Hedesiu M, Salmon B (2018) Cone-beam CT in paediatric dentistry: DIMITRA project position statement. Pediatr Radiol 48(3): 308-16.

22. Oenning AC, Pauwels R, Stratis A, De Faria Vasconcelos K, Tijskens E, De Grauwe A, et al. (2019) Halve the dose while maintaining image quality in paediatric Cone Beam CT. Sci Rep 9(1):5521.

23. Dogan MS, Callea M, Kusdhany LS, Aras A, Maharani DA, Mandasari M, et al. (2018) The evaluation of root fracture with cone beam computed tomography (CBCT): an epidemiological study. J Clin Exp Dent 10(1):e41-e8.

24. Patel S, Dawood A, Ford TP, Whaites E (2007) The potential applications of cone beam computed tomography in the management of endodontic problems. Int Endod J 40(10): 818-30.

25. May JJ, Cohenca N, Peters OA (2013) Contemporary management of horizontal root fractures to the permanent dentition: diagnosis-radiologic assessment to include cone-beam computed tomography. Pediatr Dent 35(2):120-4. 


\section{Tables}

Table 1. Number of roots with EARR diagnosed by PR, CBCT and microscopy.

\begin{tabular}{|lllll|}
\hline & \multicolumn{5}{c|}{ Number of roots } \\
\hline Diagnosis & $\mathbf{n}$ & PR & CBCT & Microscopy \\
\hline External apical root & Present & 22 & 29 & 31 \\
\hline resorption & & & & \\
\hline & Absent & 40 & 33 & \\
\hline
\end{tabular}

Table 2. True and false positives (TP, FP) and true and false negatives (TN, FN) in diagnosis of EARR.

\begin{tabular}{|llcccc|}
\hline Diagnosis & Method & TP & FP & TN & FN \\
\hline External apical root & PR & 22 & 0 & 31 & 9 \\
\hline resorption & CBCT & 29 & 0 & 31 & 2 \\
\hline Large apical root & PR & 7 & 0 & 31 & 0 \\
\hline resorption & & & & & \\
\hline CBCT & 7 & 0 & 31 & 0 \\
\hline Small apical root & PR & 15 & 0 & 31 & 9 \\
\hline resorption & & & & & \\
\hline & CBCT & 22 & 0 & 31 & 2 \\
\hline
\end{tabular}


Table 3. Sensitivity, specificity, positive predictive value (PPV), negative predictive value (NPV) and diagnostic accuracy (true positives + true negatives) for periapical radiographyy (PR) and cone-beam computed tomography (CBCT), calculated using microscopic findings as gold standard for diagnosis of EARR.

\begin{tabular}{|lllllll|}
\hline Diagnosis & Method & Sensitivity & Specificity & PPV & NPV & Accuracy \\
\hline External apical & PR & 0.70 & 1 & 1 & 0.77 & 0.85 \\
\hline root resorption & CBCT & 0.93 & 1 & 1 & 0.93 & 0.96 \\
\hline Large apical root & PR & 1 & 1 & 1 & 1 & 1 \\
\hline resorption & & & & & & \\
\hline & CBCT & 1 & 1 & 1 & 1 & 1 \\
\hline Small apical root & PR & 0.62 & 1 & 1 & 0.77 & 0.83 \\
\hline resorption & & & & & & \\
\hline & CBCT & 0.92 & 1 & 1 & 0.93 & 0.96 \\
\hline
\end{tabular}

\section{Figures}



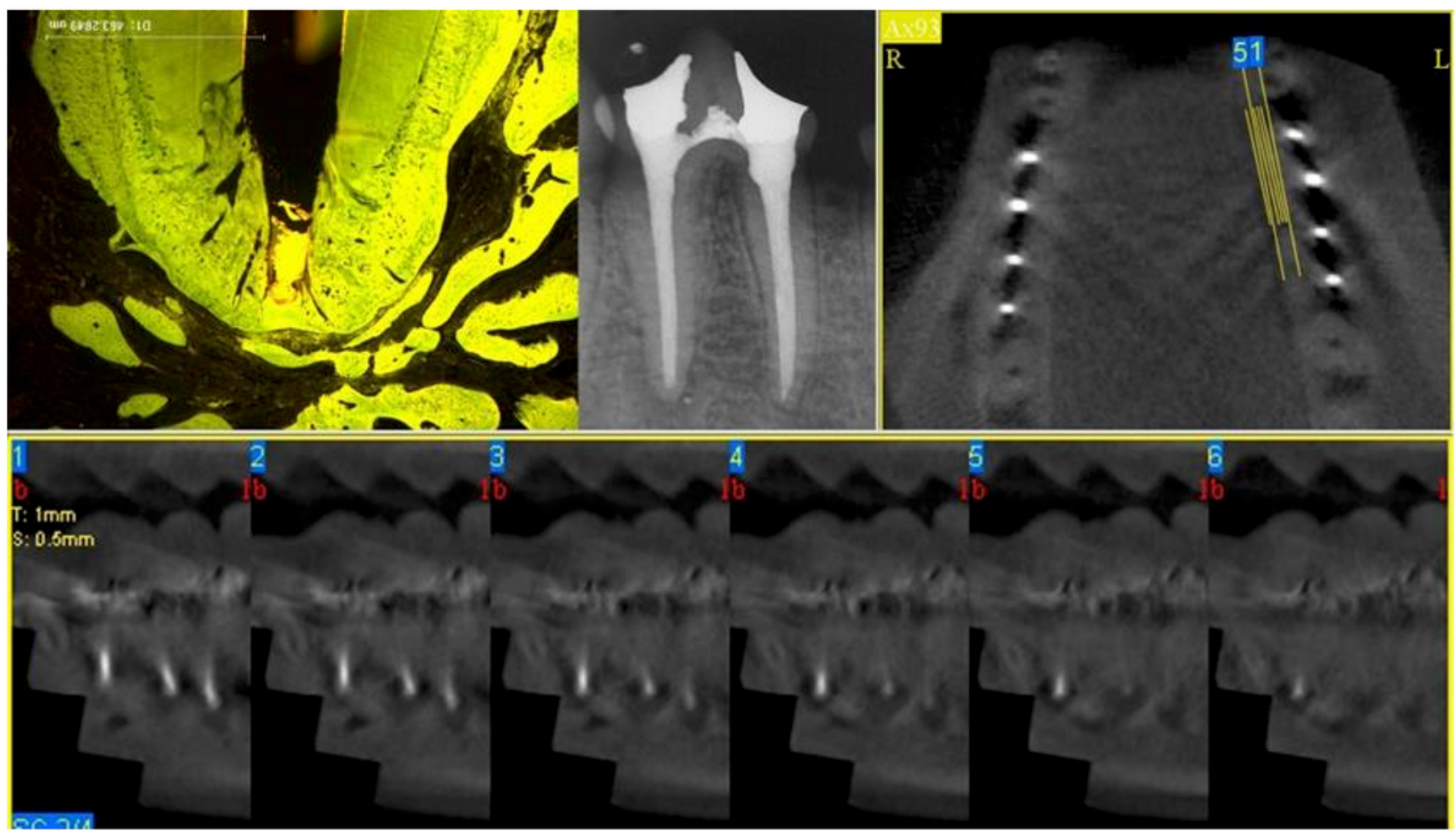

\section{Figure 1}

Representative histological view, conventional radiograph, and cone beam computed tomography (CBCT) scans from a tooth without apical root resorption. The CT scan pictures are presented in sagittal and axial views. Numbers inside blue box on sagittal views indicate the position of the section in the sequence presented on axial view. 

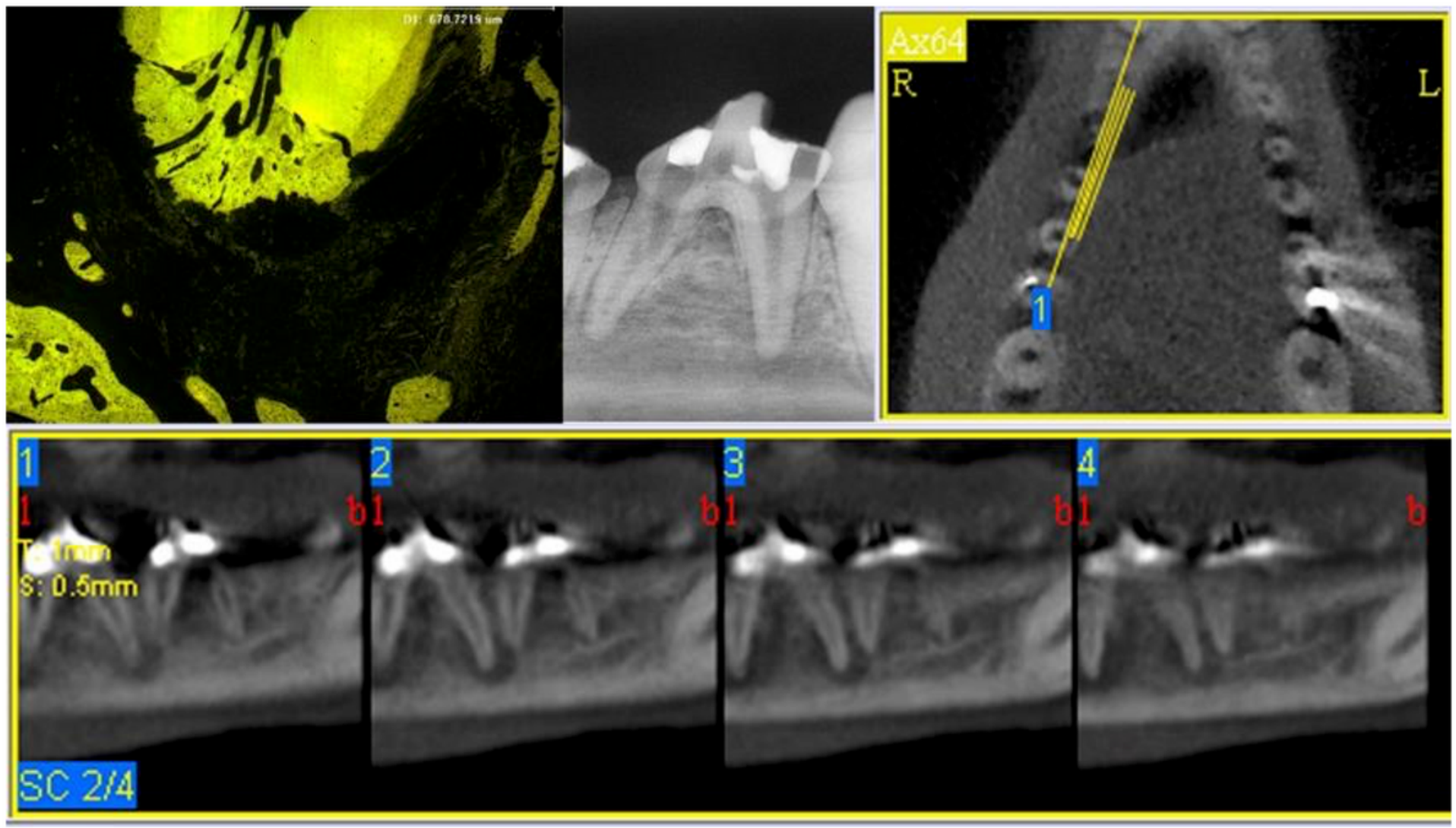

Figure 2

Representative histological view, conventional radiograph, and cone beam computed tomography (CBCT) scans from a tooth with small apical root resorption. The CT scan pictures are presented in sagittal and axial views. Numbers inside blue box on sagittal views indicate the position of the section in the sequence presented on axial view. 


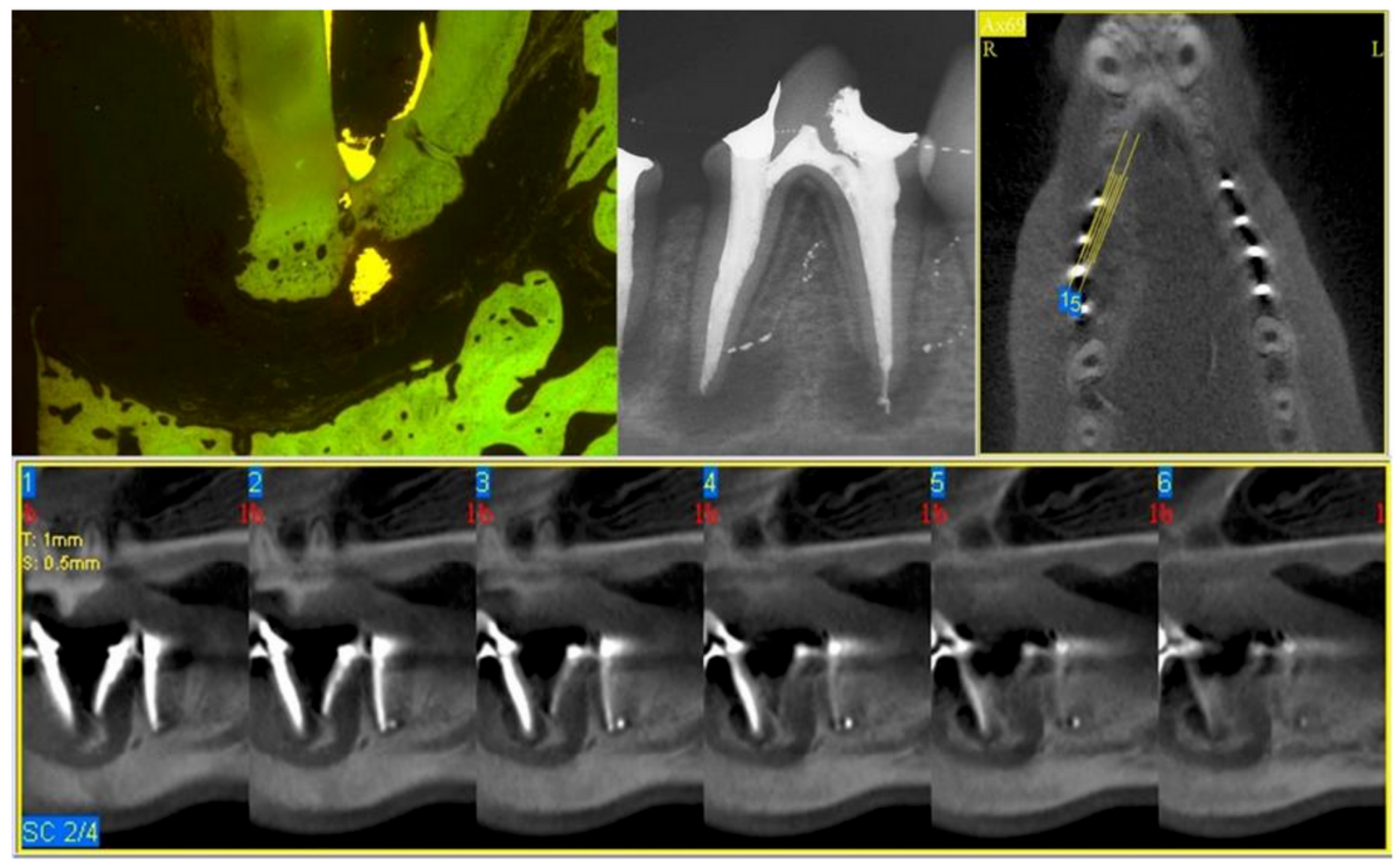

\section{Figure 3}

Representative histological view, conventional radiograph, and cone beam computed tomography (CBCT) scans from a tooth with large apical root resorption. The CT scan pictures are presented in sagittal and axial views. Numbers inside blue box on sagittal views indicate the position of the section in the sequence presented on axial view. 